\section{Aplanatic near-field optics for efficient light transfer}

\section{Doron Nakar}

Daniel Feuermann, MEMBER SPIE

Jeffrey M. Gordon*

Ben-Gurion University of the Negev

Jacob Blaustein Institutes for Desert Research

Department of Solar Energy \& Environmental Physics

Sede Boqer Campus, Israel

Abstract. Compact aplanatic concentrators can reconstitute the flux of a near-field ultrabright incoherent light source and couple it into an optical fiber. Performance near the thermodynamic limit for light transfer can be realized, even at high numerical aperture. The prospect of arc-discharge lamps as alternatives to lasers for many surgical fiber-optic procedures motivate this investigation. LED-fiber and fiberfiber coupling constitute additional applications. The contours of these achromatic mirrored systems are analytic functions, which facilitates rapid surveying of a wide range of design options. () 2006 Society of Photo-Optical Instrumentation Engineers.

[DOI: 10.1117/1.2181088]

Subject terms: optical design; optical systems.

Paper 050859L received Oct. 28, 2005; accepted for publication Jan. 9, 2006; published online Mar. 13, 2006.

Aplanatic optics that are compact and perform near the thermodynamic limit were recently developed for solar concentration (far-field sources) as well as collimation (with the roles of source and target reversed). ${ }^{1}$ Here, these solutions are generalized to the near-field problem, with illustrations of flux performance for practical applications. The motivation stems in part from the need to efficiently reconstitute the ultrabright radiant region in short-arc discharge lamps ${ }^{2-4}$ into an optical fiber, such as required for photothermal surgery, ${ }^{5,6}$ and, more generally, whenever a sizable gap between source and concentrator is imposed.

Additional applications include LED-fiber or fiber-fiber coupling. Conventional imaging devices are adequate when the numerical aperture (NA) of both source and target is small. The challenge lies in the realm of high NA, heightened by polychromatic sources because contoured refractive elements incur chromatic aberration. Accordingly, our illustrations comprise high-NA, pure-reflective optics.

The strategy is to tailor two mirror contours to eliminate spherical and comatic aberration. While the source and target NA can be chosen at will (even NA=1), imposing constraints such as low shading and blocking considerably narrows the range of admissible solutions. Figure 1 depicts the near-field problem: tracing a ray emitted at arbitrary angle $\theta$ from point source $O$ within $\mathrm{NA}_{1}=\sin \left(\theta_{\max }\right)$ to focus $F$ at angle $\phi$ within target $\mathrm{NA}_{2}=\sin \left(\phi_{\max }\right)$. Aplanats satisfy Fermat's principle of constant optical path length [Eq. (1)], as

\footnotetext{
*Also with Pearlstone Center for Aeronautical Engineering Studies, Department of Mechanical Engineering, Ben-Gurion University of the Negev, Beersheva, Israel. E-mail: jeff@bgu.ac.il

0091-3286/2006/\$22.00 @ 2006 SPIE
}

well as the Abbe sine condition, constant magnification $m$ [Eq. (2)]:

$\rho+l+r=$ const $=\rho_{o}+l_{o}+r_{o}$

$m=\sin (\theta) / \sin (\phi)=\mathrm{const}=\mathrm{NA}_{1} / \mathrm{NA}_{2}$.

Now add Snell's law and specify the distances between: (a) the source and the apex of the primary $\left(\rho_{o}\right)$; (b) the vertices of the primary and secondary $\left(l_{o}\right)$; and (c) the focus and the apex of the secondary $\left(r_{o}\right)$. Only two of these three lengths are needed since the third establishes the dimensional scale. $\mathrm{Head}^{7}$ proved that the solutions for the primary and secondary mirrors, $\rho(\theta)$ and $r(\phi)$, are analytic:

$$
\begin{aligned}
\rho(\theta)= & \ell_{o}\left\{\frac{1+k}{2 k}+\frac{1-k}{2 k} \cos (\theta)+\left[\frac{\ell_{o}}{\rho_{o}}-\frac{1}{k}\right]\right. \\
& \times\left[\frac{\gamma(\theta)}{1+m}\right]^{-1}\left[\frac{\gamma(\theta)-(1-m)}{2 m}\right]^{\alpha}\left[\frac{\gamma(\theta)-(m-1)}{2}\right]^{\beta} \\
& \left.\times\left[\frac{k+1}{2 m+2} \gamma(\theta)-\frac{k-1}{2}\right]^{2-\alpha-\beta}\right\}^{-1},
\end{aligned}
$$

where $k=\frac{\rho_{o}+r_{o}}{\ell_{o}}, \quad \alpha=\frac{m k}{m k-1}, \quad \beta=\frac{m}{m-k}$,

$$
\gamma(\theta)=\cos (\theta)+\operatorname{sign}(m) \sqrt{m^{2}-\sin ^{2}(\theta)},
$$

$$
\begin{aligned}
r(\phi)= & \ell_{o}\left\{\frac{1+k}{2 k}+\frac{1-k}{2 k} \cos (\phi)+\left[\frac{\ell_{o}}{r_{o}}-\frac{1}{k}\right]\left[\frac{\delta(\phi)}{1+M}\right]^{-1}\right. \\
& \times\left[\frac{\delta(\phi)+M-1}{2 M}\right]^{\alpha^{\prime}}\left[\frac{\delta(\phi)+1-M}{2}\right]^{\beta^{\prime}} \\
& \left.\times\left[\frac{k+1}{2 M+2} \delta(\phi)-\frac{k-1}{2}\right]^{2-\alpha^{\prime}-\beta^{\prime}}\right\}^{-1},
\end{aligned}
$$

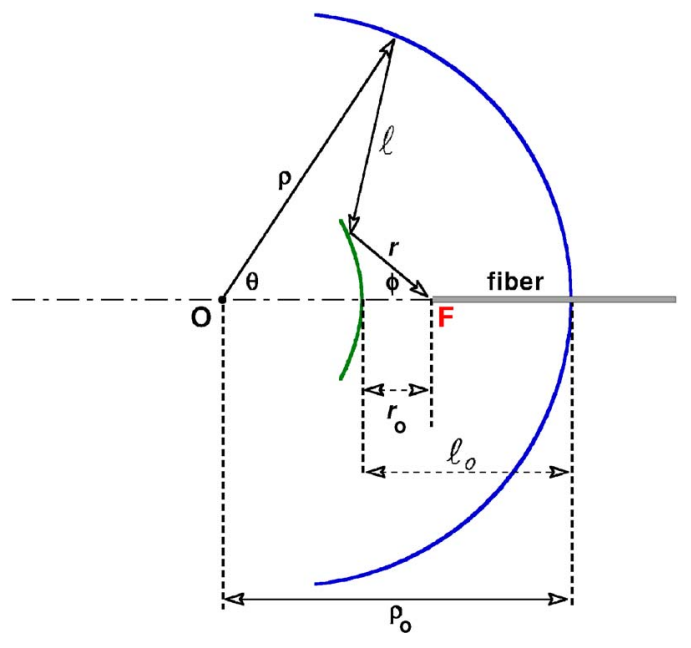

Fig. 1 Aplanatic design. Rays are focused from a source at point $O$ to a target $F$ (e.g., the tip of an optical fiber). 

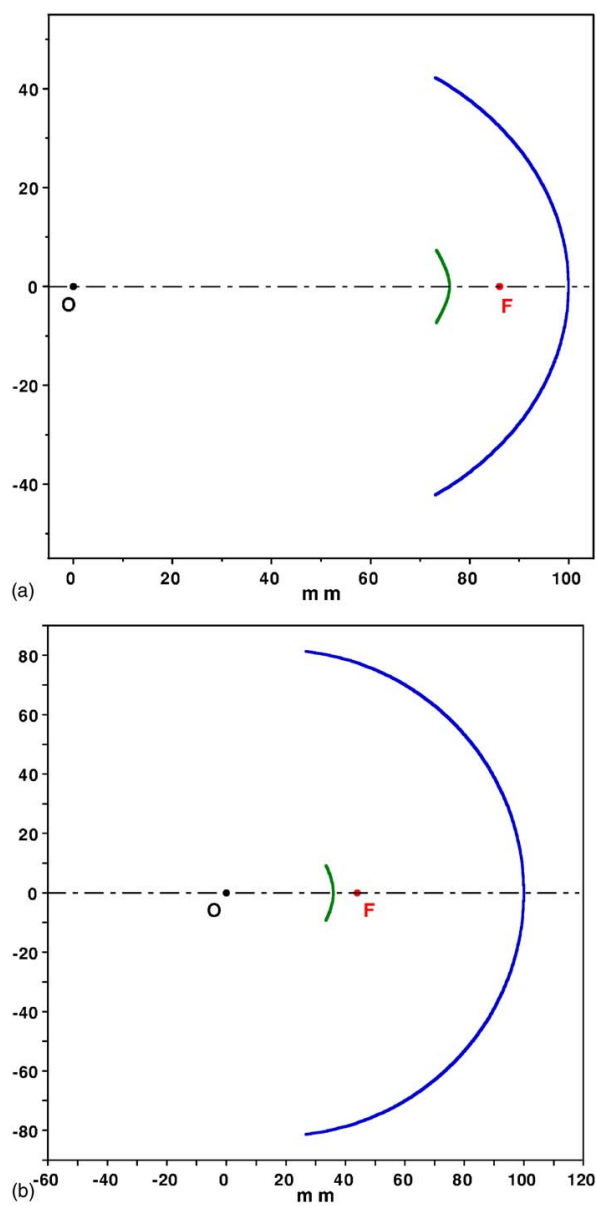

Fig. 2 Two illustrative designs. $\rho_{o}=100 \mathrm{~mm}$ sets the length scale. (a) $I_{o}=24 \mathrm{~mm}, r_{o}=10 \mathrm{~mm}, \mathrm{NA}_{1}=\mathrm{NA}_{2}=0.50$, shading $=0.040$. (b) $I_{o}$ $=64 \mathrm{~mm}, r_{o}=8 \mathrm{~mm}, \mathrm{NA}_{1}=0.95, \mathrm{NA}_{2}=0.66$, shading $=0.079$.

where $\alpha^{\prime}=\frac{M k}{M k-1}, \quad \beta^{\prime}=\frac{M}{M-k}, \quad M=1 / m$,

$\delta(\phi)=\cos (\phi)+\operatorname{sign}(M) \sqrt{M^{2}-\sin ^{2}(\phi)}$.

Figure 2 presents two sample designs: Fig. 2(a) is prompted by LED-fiber and fiber-fiber coupling, and Fig. 2(b) is motivated by high-flux irradiation of an optical fiber from a short-arc discharge lamp or high-NA LED. For the discharge lamp, $\mathrm{NA}_{1}=0.95$ refers to emission into the hemisphere facing the fiber, and incorporates occlusion of emitted light by the lamp's electrodes. ${ }^{2-4} \mathrm{NA}_{2}=0.66$ represents the highest NA available in commercial fibers that are transmissive over the visible and near-IR. ${ }^{8}$ The concentrators are ultracompact (aspect ratios in the range 0.3 to 0.5 ).

Earlier studies of aplanats ${ }^{7,9}$ dealt with diverging systems only, where the caustic from the primary lies to the left of (beyond) the secondary (Figs. 1 and 2 are diverging designs). A complementary class of converging solutions exists: the caustic from the primary lies between the two mirrors (Fig. 3). The analytic solutions for the mirror contours [Eq. (3)] are the same, but with $\phi<0$, hence negative $\mathrm{NA}_{2}$ and $m$. No advantages were apparent for the converging aplanats relative to their diverging counterparts.

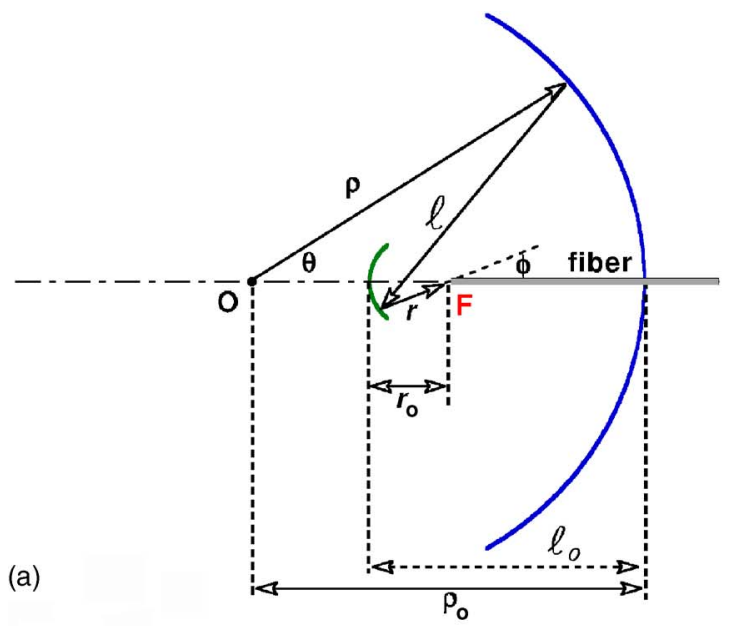

(b)

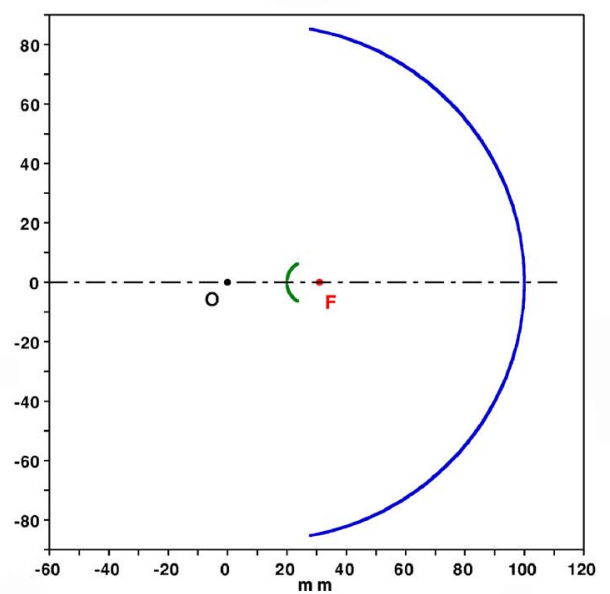

Fig. 3 (a) Converging design, where $\phi, \mathrm{NA}_{2}$, and $m$ are negative. (b) Converging complement to Fig. 2(b), with $\mathrm{NA}_{2}=-0.66, \mathrm{NA}_{1}$ $=0.95, \rho_{o}=100 \mathrm{~mm}, I_{o}=80 \mathrm{~mm}, r_{o}=11 \mathrm{~mm}$, shading $=0.074$.

Performance of the high-NA optical systems of Fig. 2 was evaluated by commercial raytrace simulation. 250,000 rays evenly distributed in phase space were traced from an extended source centered at $O$ to a target disk centered at $F$. Material-specific losses not included are: (a) mirror absorp-

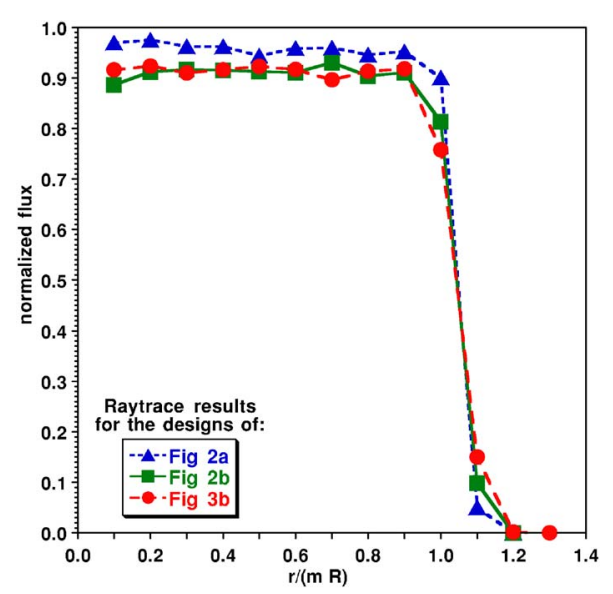

Fig. 4 Flux, normalized to source flux, as a function of nondimensional target radial position, for disk-to-disk transfer. 

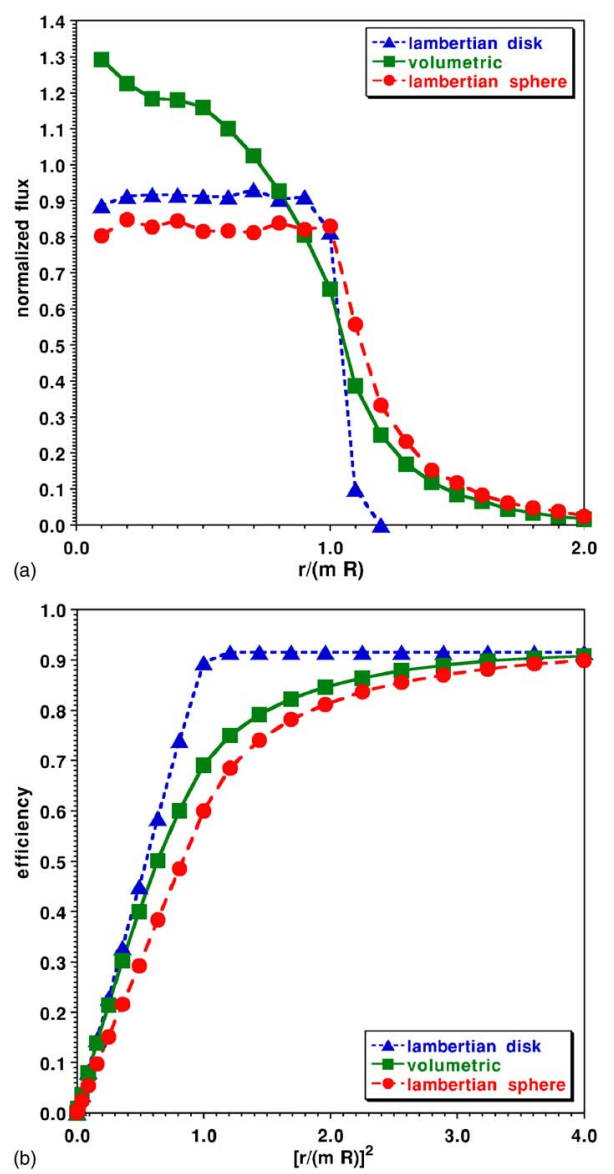

Fig. 5 (a) Sensitivity of flux map to source geometry. Design of Fig. 2(b) ( $m=1.44$, disk target). Flux here is normalized to $1 / m^{2}$ of source power density. The source subtends an angular radius of $6 \mathrm{mrad}$ at the rim of the primary. The nonuniform flux map at low $r$ with the volumetric source stems from its being nonlambertian. (b) Efficiency vs nondimensionalized target area, accounting for shading, blocking, and ray rejection.

tion $1-\Omega^{2}$ ( $\Omega=$ reflectivity) with each ray undergoing two reflections, and (b) Fresnel reflections at the fiber.

Three sources were considered: (1) a lambertian disk (germane to LED-fiber and fiber-fiber coupling), (2) a lambertian sphere, and (3) a spherical volumetric source composed of uniformly distributed point sources. The volumetric source is brighter than the lambertian sphere and disk at small emission angles due to its brighter core region (i.e., it is closer to a point source). This explains why the flux near the target's center should exceed that generated with either a lambertian sphere or disk of the same radius.

The flux map in the focal plane was generated for a source radius $R=0.5 \mathrm{~mm}$. Results for disk-to-disk transfer are plotted in Fig. 4. An étendue-matched system is a useful reference point, with $r=m R$ being the smallest target radius consistent with the constrained thermodynamic limit. ${ }^{1,10}$ Rays that miss either the secondary or the target constitute only 0.011 and 0.021 of the radiation entering the primary for the aplanats of Figs. 2(a) and 2(b), respectively, and 0.032 for the complementary design of Fig. 3(b).

Optical performance is quantified in Fig. 5. Efficiency

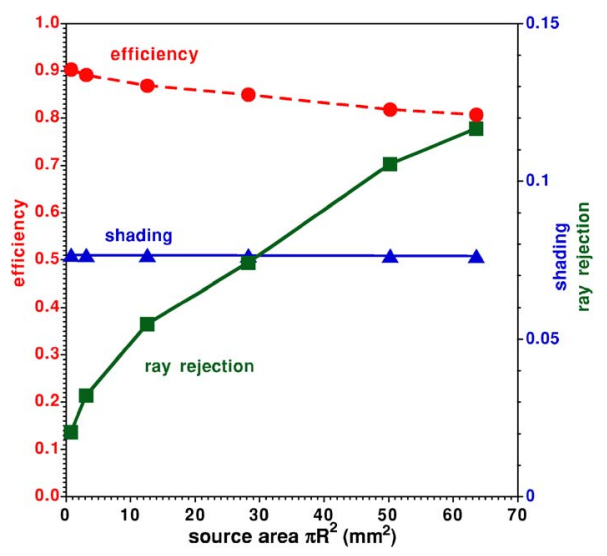

Fig. 6 Efficiency and individual loss terms as functions of source area for disk-to-disk transfer in an étendue-matched design of Fig. 2(b). For source areas up to $45 \mathrm{~mm}^{2}$, corresponding to an angular radius up to $22 \mathrm{mrad}$ at the rim of the primary (more than 3 times that modeled in Figs. 4 and 5), blocking is less than $1 \%$ and ray rejection remains below $10 \%$.

refers to the power within a given target area relative to the power impinging on the primary. For a discharge lamp, if only a single fiber or concentrator is required, lamp emissions could be recycled back to the source, ${ }^{4}$ e.g., with a hemispherical mirror. ${ }^{5}$ Increasing source size amplifies higher-degree aberrations and worsens optical performance, as summarized in Fig. 6.

The near-field aplanats presented here do not incur chromatic aberration. Design and optimization studies can be rapid and precise because the mirror contours have analytic solutions. Applications in light-based surgery, LED-fiber coupling, and fiber-fiber coupling have motivated this investigation. Although aplanats have long been recognized, ${ }^{7,9}$ their performance as flux transformers had not been explored. We have expanded the far-field cases recently analyzed ${ }^{1}$ to near-field, and shown they can meet the challenge of high-NA systems.

\section{References}

1. J. M. Gordon and D. Feuermann, "Optical performance at the thermodynamic limit with tailored imaging designs," Appl. Opt. 44, 2327-2331 (2005).

2. Hamamatsu, Shimokanzo, Iwata-gun, Shizuoka-ken, 438-0193, Japan, technical brochures and private communications (2004).

3. U. Weichmann, J. W. Cromwijk, G. Heusler, U. Mackens, H. Moench, and J. Pollman-Retsch, "Light sources for small-étendue applications," Proc. SPIE 5740, 13-26 (2005).

4. U. Weichmann, H. Giese, U. Hechtfischer, G. Heusler, A. Koerber, H. Moench, F. C. Noertemann, P. Pekarski, J. Pollman-Retsch, and A. Ritz, "UHP lamps for projection systems," Proc. SPIE 5289, 255-265 (2004).

5. D. Nakar, D. Feuermann, and J. M. Gordon, "Near-field tailored imaging optics for coupling ultra-bright light sources into optical fibers," Proc. SPIE 5942, 171-178 (2005).

6. D. Feuerman, J. M. Gordon, and T. W. Ng, "Photonic surgery with noncoherent light," Appl. Phys. Lett. (in press).

7. A. K. Head, "The two-mirror aplanat," Proc. Phys. Soc. London, Sect. B 70, 945-949 (1957)

8. Polymicro Technologies LLC, 18019 N. 25th Ave., Phoenix, AZ, technical prospectus and private communications (2001).

9. K. Schwarzschild, "Untersuchungen zur geometrischen Optik I-III," Abh. Konigl. Ges. Wis. Gottingen Math-phys. Kl. 4, Nos. 1-3 (19051906) (in German).

10. R. Winston, J. C. Minano, and P. Benitez, Nonimaging Optics, Elsevier Academic Press, Oxford (2005). 